\title{
Prevalence and Significance of FDG-avid Mediastinal Lymph Nodes in Patients with Colorectal Cancer in a Tuberculosis-endemic Area
}

\author{
Yutapong Raruenrom, M.D. ${ }^{1}$, Daris Theerakulpisut, M.D. ${ }^{1}$, Mix Wannasarnmetha, M.D. ${ }^{2}$ \\ 'Division of Nuclear Medicine, Department of Radiology, Faculty of Medicine, Khon Kaen University, Mueang, Khon Kaen 40002 , \\ Thailand. \\ ${ }^{2}$ Srinagarind Center of Excellence for Neurovascular Intervention and Surgery, Faculty of Medicine, Khon Kaen University, \\ Mueang, Khon Kaen 40002, Thailand. \\ Received 30 April 2021 • Revised 20 June 2021 • Accepted 20 June 2021 • Published online 1 September 2021
}

\section{Abstract:}

Objective: To find prevalence and causes of fluorodeoxyglucose (FDG)-avid mediastinal lymph nodes in patients with colorectal cancer in a tuberculosis-endemic area.

Material and Methods: For this study we enrolled patients with colorectal cancer who underwent Fluorine-18 fluorodeoxyglucose positron emission tomography/computed tomography (F-18 FDG PET/CT). Then, PET/CT images were reviewed by a nuclear medicine physician to find mediastinal lymph nodes with FDG uptake beyond the lung background defined as FDG-avid node. The degree of FDG uptake was graded and measured, and associated factors for presence of FDG-avid nodes were evaluated. The causes of FDG-avid nodes were also determined.

Results: A total of 109 patients (64 males; mean age 61.2 years) were studied. Seventy-five patients had FDG-avid mediastinal nodes; accounting for a prevalence of $68.8 \%(95 \% \mathrm{Cl}: 59.2-77.3 \%)$. Most of the patients had multiple and bilateral nodes; with the zones of hilar and interlobar being the most common location. Age $\geq 50$ years was the only associated factor for FDG-avid nodes (OR of 3.16, p-value=0.035). Only one out of the 32 patients (with fulfilled followup criteria) had a metastatic node.

Conclusion: The prevalence of FDG-avid mediastinal nodes in colorectal cancer patients in a tuberculosis-endemic area was significantly high. Most of the lesions were benign in nature; thus, interpretation of these findings should be considered carefully to avoid false-positive results.

Keywords: colorectal cancer, F-18 FDG PET/CT, mediastinal lymph node, tuberculosis-endemic area

Contact: Yutapong Raruenrom, M.D.

Division of Nuclear Medicine, Department of Radiology, Faculty of Medicine,

Khon Kaen University, Mueang, Khon Kaen 40002, Thailand.

E-mail: yutara@kku.ac.th

This is an open access article under the CC BY-NC-ND license

(http://www.jhsmr.org/index.php/jhsmr/about/editorialPolicies\#openAccessPolicy).

J Health Sci Med Res 2022;40(3):261-270 doi: $10.31584 /$ jhsmr.2021833 www.jhsmr.org 


\section{Introduction}

Colorectal cancer (CRC) is the fourth most common cancer; after lung, breast, and prostate malignancies, and accounts for approximately $10.0 \%$ of all cancerrelated deaths worldwide. ${ }^{1}$ Male gender, increasing age, and family history are significant risk factors for disease incidence. $^{2}$ Metastatic disease was observed in $20.0 \%$ of patient diagnoses. The most common distant metastatic site is the liver (more than 60.0\%), followed by the lung, peritoneum, bone, and the brain. ${ }^{3}$ Mediastinal lymph node metastasis from CRC, classified as distant metastasis, is rare and usually found as a metachronous metastasis. The route of lymphatic drainage from the primary tumor to the mediastinum is still unclear. A possible mechanism is the reflux of tumor emboli from the thoracic duct into the bronchopulmonary trunk. ${ }^{4,5}$ Many reports have shown a better prognosis with low disease recurrent rates in patients with resectable mediastinal lymph node metastasis. ${ }^{5-8}$

Fluorine-18 fluorodeoxyglucose positron emission tomography/computed tomography (F-18 FDG PET/CT) plays an appropriate role for detection of local recurrence or metastatic $\mathrm{CRC}$, in the case of rising tumor markers with negative or equivocal first-line imaging. The pooled sensitivity and specificity of PET/CT for detection of recurrent distant metastasis were 91.0-94.0\% and 77.0$93.0 \%$, respectively. ${ }^{9}$ However, the accuracy of F-18 FDG PET/CT for detection of mediastinal lymph node metastasis can be lower in a tuberculosis-endemic area. This is because of increased glucose metabolism by activated macrophages and inflammatory phagocytes in the granulomatous diseases, which cause a false-positive scan. ${ }^{10,11}$ Thailand is a tuberculosis-endemic area, and most of the patients $(80.0 \%)$ had pulmonary tuberculosis (TB). ${ }^{12}$ Thus, there is a possibility of having more patients with FDG-avid mediastinal lymph nodes here than in Western countries that may be difficult to interpret; especially in CRCs which had rare mediastinal lymph node metastasis.
However, to the best of our knowledge, there is no report concerning prevalence and significance of FDG-avid mediastinal lymph nodes in patients with CRC within this area. Hence, we primarily aimed to find a prevalence of FDG-avid mediastinal lymph nodes in patients with CRC in a tuberculosis-endemic area. Secondly, we also aimed to evaluate the causes and associated factors of FDG-avid mediastinal lymph nodes in this group of patients.

\section{Material and Methods}

This retrospective descriptive study was approved by the Khon Kaen University Ethics Committee for Human Research (Reference number: HE631059), and the requirement for informed consent was waived. From September 2013 to January 2020, patients with colorectal cancer who underwent F-18 FDG PET/CT at Srinagarind Hospital were consecutively enrolled. Patients who had second primary malignancy were excluded from the study.

\section{F-18 FDG PET/CT protocol}

All patients fasted at least 6 hours prior to the scan. $\mathrm{PET} / \mathrm{CT}$ was performed by $3 \mathrm{D}$ time-of-flight mode, using Discovery PET/CT 690 (GE Healthcare). The uptake time was 60 minutes after intravenous injection of 5.18-7.40 MBq (0.14-0.20 mCi) per kilogram of F-18 fluorodeoxyglucose (F-18 FDG). PET/CT images were obtained from vertexto-mid-thigh in the supine position. Emission images were acquired while within normal tidal respiration, with a speed of 2-3 minutes per bed position after contrast-enhanced 64-slice CT (120 kVp, automatic mA, $3.75 \mathrm{~mm}$ slice thickness).

\section{F-18 FDG PET/CT interpretation}

$\mathrm{PET} / \mathrm{CT}$ images were reviewed by a nuclear medicine physician. FDG-avid mediastinal lymph node was defined as a mediastinal lymph node with FDG uptake beyond the lung background. The degree of FDG avidity was graded 
by visual inspection; as per the following details: Grade 1: Mediastinal lymph node uptake < blood pool activity, Grade 2: Blood pool activity $\leq$ mediastinal lymph node uptake < liver activity, Grade 3: Mediastinal lymph node uptake $\geq$ liver activity. The semi-quantitative assessment of FDG-avid lymph node was evaluated by the maximum standardized uptake values $\left(S U V_{\max }\right)$ of the lymph nodes, with maximal uptake in each patient and the lymph node-to-liver ratio (LLR). A spherical 3D region of interest (ROI) was drawn around the lymph node on the axial images and used to calculate the SUV $\max$. For an average standardized uptake value (SUV $\mathrm{av}_{\text {) }}$ of the liver, spherical 3D ROI with a diameter of approximately $3 \mathrm{~cm}$ was placed on the normal inferior right lobe of the liver. The LLR was a ratio of SUV $\max$ of lymph node to SUV av of the liver.

The location of any FDG-avid lymph node was evaluated according to the International Association of the Study of Lung Cancer (IASLC) lymph node map, by excluding the supraclavicular zone because of its potential metastatic site of CRC. Size of the lymph node was measured in the short-axis diameter. CT characteristics of the lymph nodes (shape and calcification) were also assessed. Other hypermetabolic tumor lesions were also reviewed.

Lung parenchymal lesions were evaluated by a diagnostic radiologist, who was blinded to the clinical information.

\section{Causes of FDG-avid mediastinal lymph nodes}

The benign lymph nodes were defined as negative for malignancy by biopsy, or stable/ decreased in size of the lymph nodes on the serial diagnostic chest CT or $\mathrm{PET} / \mathrm{CT}$ over 6 months. Determination of metastatic lymph nodes was performed by using histopathological results, or evidence of an increase in the size of the nodes of more than $20.0 \%$; with absolute increase of more than $5 \mathrm{~mm}$ on the serial images.
The prevalence of FDG-avid mediastinal lymph nodes was calculated based on per-patient analysis. The categorical data is presented as numbers and percentages. The continuous data is presented as mean \pm standard deviation (S.D.) or median (interquartile range). A comparison of demographic data in patients with the presence and absence of FDG-avid lymph nodes was performed using chi-square and Fisher's exact Test. Univariable analysis by bivariate logistic regression was used to test the association between variables (age, gender, tumor location, lung parenchymal lesion, hypermetabolic tumor lesion) and presence of FDG-avid mediastinal lymph nodes. The multivariable analysis by multiple logistic regression was used to calculate the adjusted odds ratios. Kruskal-Wallis Test was used to compare median SUV $_{\max }$ and LLR of the lymph nodes in each grade. The $95 \%$ confidence interval $(95 \% \mathrm{Cl})$ was calculated where appropriated. All statistical methods were two-sided, and any $p$-value of less than 0.050 were considered statistically significant. Statistical analysis was carried out using STATA 10.1 (StataCorp LP, College Station, TX, USA).

\section{Results}

There were 119 patients with colorectal cancer having undergone F-18 FDG PET/CT between September 2013 and January 2020. Five patients with second primary cancer and five without available PET/CT images were excluded. Thus, there were a total of 109 analyzed patients. Seventyfive patients had FDG-avid mediastinal lymph nodes, which accounted for a prevalence of $68.8 \%(95 \% \mathrm{Cl}: 59.2-77.3 \%)$. The causes of FDG-avid lymph nodes were identified in 32 patients (42.7\%). Detailed patient selection and follow-up is presented in Figure 1.

The mean age of patients was $61.2 \pm 11.4$ years. Most of them were aged 50 years or above ( $n=91,83.5 \%)$, with more than half being male $(n=64,58.7 \%)$. The primary tumor locations were at the colon $(n=73,67.0 \%)$ and rectum $(n=$ 
36, 33.0\%), respectively. PET/CT was commonly requested for detection of recurrent disease $(n=68,62.4 \%)$. More than half of the patients had lung parenchymal lesions which included nodules and interstitial infiltration ( $n=62,56.9 \%)$ as well as hypermetabolic tumor lesions ( $n=75,68.8 \%)$. A comparison of demographic data in patients with and without FDG-avid mediastinal lymph nodes is shown in
Table 1. There was a significant difference between the age of patients with and without FDG-avid mediastinal lymph nodes $(p-v a l u e=0.015)$. However, there were no significant differences between gender, tumor location, PET/ CT indications, presence of lung lesions, and presence of hypermetabolic tumor lesions, between the two groups.

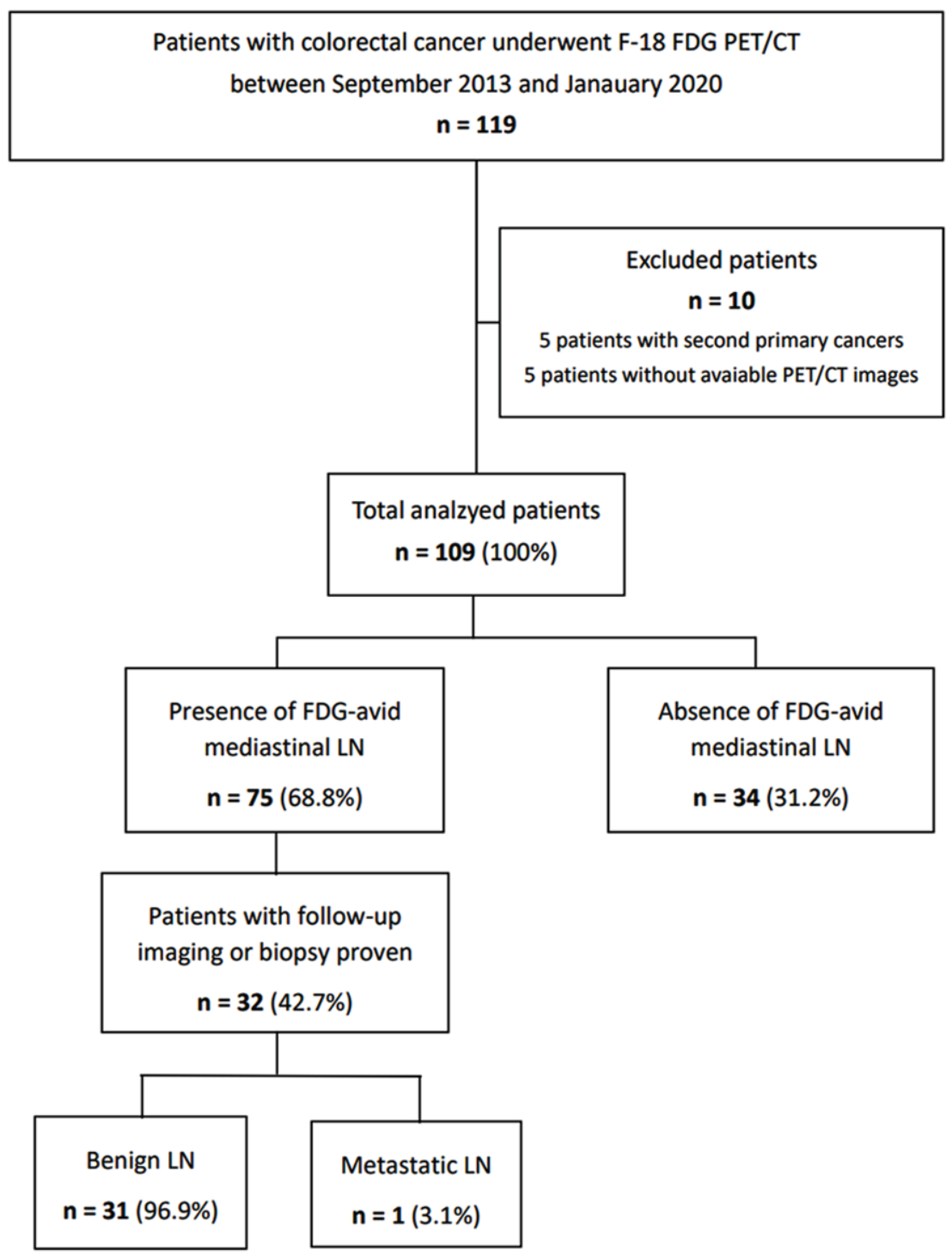

F-18 FDG PET/CT=fluorine-18 fluorodeoxyglucose positron emission tomography/computed tomography, FDG=fluorodeoxyglucose, $\mathrm{LN}=$ lymph node

Figure 1 Flowchart of patient selection and study 
Table 1 Patient's demographic data

\begin{tabular}{|c|c|c|c|c|}
\hline \multirow[b]{2}{*}{ Characteristics } & \multicolumn{2}{|c|}{ FDG-avid mediastinal LN } & \multirow[b]{2}{*}{$p$-value } & \multirow{2}{*}{$\begin{array}{l}\text { Total }(\%) \\
109(100)\end{array}$} \\
\hline & $\begin{array}{l}\text { Presence (\%) } \\
75(68.8)\end{array}$ & $\begin{array}{l}\text { Absence (\%) } \\
34(31.2)\end{array}$ & & \\
\hline Age (years) & & & $0.015^{\star}$ & \\
\hline$<50$ & $8(10.7)$ & $10(29.4)$ & & $18(16.5)$ \\
\hline$\geq 50$ & $67(89.3)$ & $24(70.6)$ & & $91(83.5)$ \\
\hline Sex & & & 0.686 & \\
\hline Male & $45(60.0)$ & $19(55.9)$ & & $64(58.7)$ \\
\hline Female & $30(40.0)$ & $15(44.1)$ & & $45(41.3)$ \\
\hline Tumor location & & & 0.589 & \\
\hline Colon & $49(65.4)$ & $24(70.6)$ & & $73(67.0)$ \\
\hline Rectum & $26(34.6)$ & $10(29.4)$ & & $36(33.0)$ \\
\hline Indication for F-18 FDG PET/CT & & & 0.497 & \\
\hline Detection of recurrent disease & $46(61.3)$ & $22(64.7)$ & & $68(62.4)$ \\
\hline Initial staging & $4(5.3)$ & $4(11.8)$ & & $8(7.3)$ \\
\hline Evaluation of treatment response & $24(32.0)$ & $8(23.5)$ & & $32(29.4)$ \\
\hline Other & $1(1.4)$ & 0 & & $1(0.9)$ \\
\hline Lung parenchymal lesion & & & 0.163 & \\
\hline Presence & $46(61.3)$ & $16(47.1)$ & & $62(56.9)$ \\
\hline Absence & $29(38.7)$ & $18(52.9)$ & & $47(43.1)$ \\
\hline Hypermetabolic tumor lesions & & & 0.474 & \\
\hline Presence & $50(66.7)$ & $25(73.5)$ & & $75(68.8)$ \\
\hline Absence & 25 (33.3) & $9(26.5)$ & & $34(31.2)$ \\
\hline
\end{tabular}

*statistically significant

F-18 FDG PET/CT=fluorine-18 fluorodeoxyglucose positron emission tomography/computed tomography, FDG=fluorodeoxyglucose,

LN=lymph node

Table 2 Details of fluorodeoxyglucose-avid mediastinal lymph nodes, based on per-patient analysis

\begin{tabular}{ll}
\hline Characteristics & Number (\%) \\
\hline Number & $5(6.7)$ \\
Single & $70(93.3)$ \\
Multiple & \\
Laterality & $12(16.0)$ \\
Unilateral & $63(84.0)$ \\
Bilateral & \\
Location & $59(78.7)$ \\
Upper zone & $32(42.7)$ \\
Aortopulmonary zone & $42(56.0)$ \\
Subcarinal zone & $7(9.3)$ \\
Lower zone & $68(90.7)$ \\
Hilar and interlobar zone & 0 \\
Peripheral zone & \\
Grade of uptake & $8(10.7)$ \\
Grade 1 & $42(56.0)$ \\
Grade 2 & $25(33.3)$ \\
Grade 3 & $0.95 \pm 0.25$ \\
Mean size in cm \pm S.D. & \\
Shape & $53(70.7)$ \\
Oval & $22(29.3)$ \\
Round & \\
Internal calcification & $10(13.3)$ \\
Yes & $65(86.7)$ \\
No &
\end{tabular}

Among the 75 patients with FDG-avid mediastinal lymph nodes, 70 patients (93.3\%) had multiple nodes and 63 patients $(84.0 \%)$ had bilateral nodes. The common three lymph node locations were at within the hilar and interlobar zones ( $n=68,90.7 \%)$, upper zone $(n=59,78.7 \%)$, and subcarinal zone $(n=42,56.0 \%)$, respectively. The mean size of lymph nodes was $0.95 \pm 0.25 \mathrm{~cm}$. The details of FDG-avid mediastinal lymph nodes are shown in Table 2.

Regarding the degree of FDG avidity, more than half of the patients ( $n=42,56.0 \%$ ) had grade 2 , with median SUV $_{\max }$ of 4.4 (IQR: 4.2, 4.9) and median LLR of 1.8 (IQR: $1.7,2.1)$. About one-third of patients $(n=25,33.3 \%)$ had intense uptake (grade 3) median SUV max $_{\text {of }} 7.3$ (IQR: 6.7, 8.5) and median LLR of 3.5 (IQR: 2.6, 3.8). Eight patients $(10.7 \%)$ had mild uptake (grade 1) with median SUV $V_{\max }$ of 2.95 (IQR: 2.6, 3.1) and median LLR of 1.2 (IQR: 1.1, 1.4). There was a significant difference between median SUV $V_{\max }$ and LLR of lymph nodes in each grade ( $p$-value<0.010). 
Multivariable analysis showed a significant association between patients aged 50 years or above, and presence of FDG-avid mediastinal lymph nodes (adjusted OR of 3.16, 95\% Cl: 1.09-9.22, p-value=0.035). However, there was no significant association between gender, primary tumor location, lung parenchymal lesion, and hypermetabolic tumor lesion; as shown in Table 3.

Table 4 shows causes of FDG-avid mediastinal lymph nodes in 32 patients having had biopsy proven or serial images. Almost all these patients had benign lymph nodes $(n=31,96.9 \%)$, based on their histopathological results and follow-up images. Almost half in this group (15 patients) had grade 2 uptake, while 13 and 3 patients had grade 3 and grade 1 uptake, respectively. One patient with metastatic FDG-avid lymph node (Grade 3 uptake, SUV $V_{\max }$ of 6.6, LLR of 2.2) was determined by a follow-up diagnostic chest CT, which showed a significant increase in size of the right paratracheal lymph node; from 1.2 to 2.5 $\mathrm{cm}$, with internal necrosis. The example cases of benign and metastatic lymph nodes are demonstrated in Figure 2 and Figure 3, respectively.

Table 3 Association between variables and presence of fluorodeoxyglucose-avid mediastinal lymph nodes

\begin{tabular}{|c|c|c|c|c|}
\hline \multirow{2}{*}{ Variable } & \multicolumn{2}{|c|}{ Univariable analysis } & \multicolumn{2}{|c|}{ Multivariable analysis } \\
\hline & OR $(95 \% \mathrm{Cl})$ & $p$-value & Adjusted OR $(95 \% \mathrm{Cl})$ & $p$-value \\
\hline \multicolumn{5}{|l|}{ Age (years) } \\
\hline$<50$ & 1 & & 1 & \\
\hline$\geq 50$ & $3.49(1.23-9.87)$ & $0.019^{*}$ & $3.16(1.09-9.22)$ & $0.035^{*}$ \\
\hline \multicolumn{5}{|l|}{ Sex } \\
\hline Female & 1 & & 1 & \\
\hline Male & $1.18(0.52-2.69)$ & 0.686 & $0.94(0.39-2.25)$ & 0.893 \\
\hline \multicolumn{5}{|c|}{ Location of primary tumor } \\
\hline Colon & 1 & & 1 & \\
\hline Rectum & $1.27(0.53-3.06)$ & 0.589 & $1.12(0.45-2.81)$ & 0.803 \\
\hline \multicolumn{5}{|c|}{ Lung parenchymal lesion } \\
\hline Absence & 1 & & 1 & \\
\hline Presence & $1.78(0.78-4.04)$ & 0.165 & $1.71(0.72-4.08)$ & 0.222 \\
\hline \multicolumn{5}{|c|}{ Hypermetabolic tumor lesion } \\
\hline Absence & 1 & & 1 & \\
\hline Presence & $0.72(0.29-1.77)$ & 0.475 & $0.71(0.28-1.83)$ & 0.480 \\
\hline
\end{tabular}

*statistically significant

Table 4 Causes of fluorodeoxyglucose-avid mediastinal lymph nodes in 32 patients

\begin{tabular}{ll}
\hline Causes & Number (\%) \\
\hline Benign & $31(96.9)$ \\
By biopsy proven & $2(6.2)$ \\
By follow-up chest CT & $23(71.9)$ \\
By follow-up FDG PET/CT & $6(18.8)$ \\
\hline Metastasis by follow-up chest CT & $1(3.1)$ \\
\hline
\end{tabular}

$\mathrm{CT}=$ computed tomography, FDG PET/CT=fluorodeoxyglucose positron emission tomography/computed tomography 

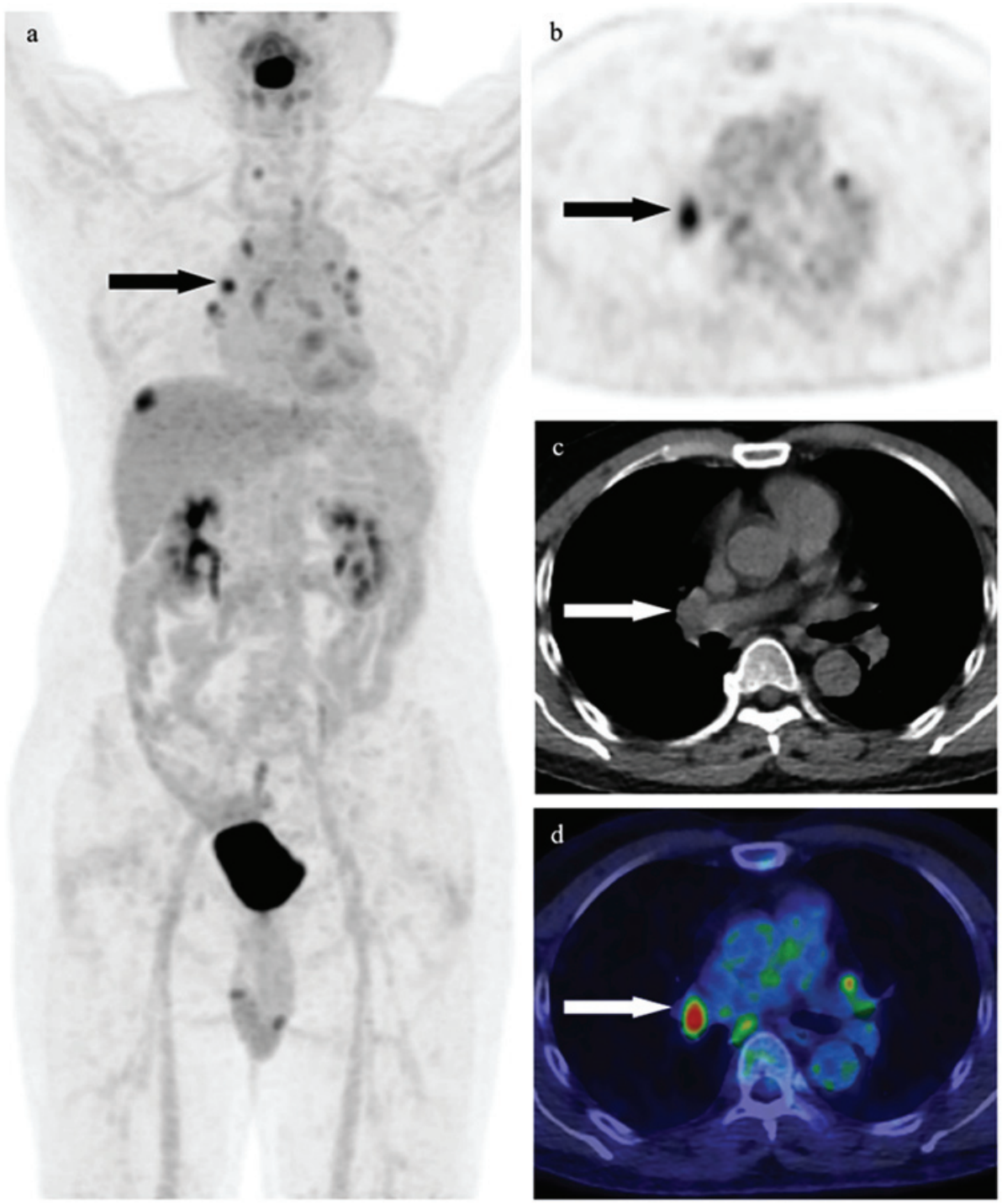

Figure 2 Benign lymph node: A 64-year-old male with stage IV colon cancer; with liver metastasis status post right hemicolectomy, with adjuvant chemotherapy underwent positron emission tomography/computed tomography for evaluation of treatment response. Fluorine-18 fluorodeoxyglucose positron emission tomography/computed tomography showed multiple fluorodeoxyglucose-avid mediastinal lymph nodes. The most intense lymph node was sized at $1.2 \mathrm{~cm}$ and is at the right hilar region with maximum standardized uptake values of 9.0 and lymph node-to-liver ratio of 3.5 (2a, 2b, 2c, 2d). The lymph node biopsy showed reactive hyperplasia, with a large amount of anthracotic pigment. 

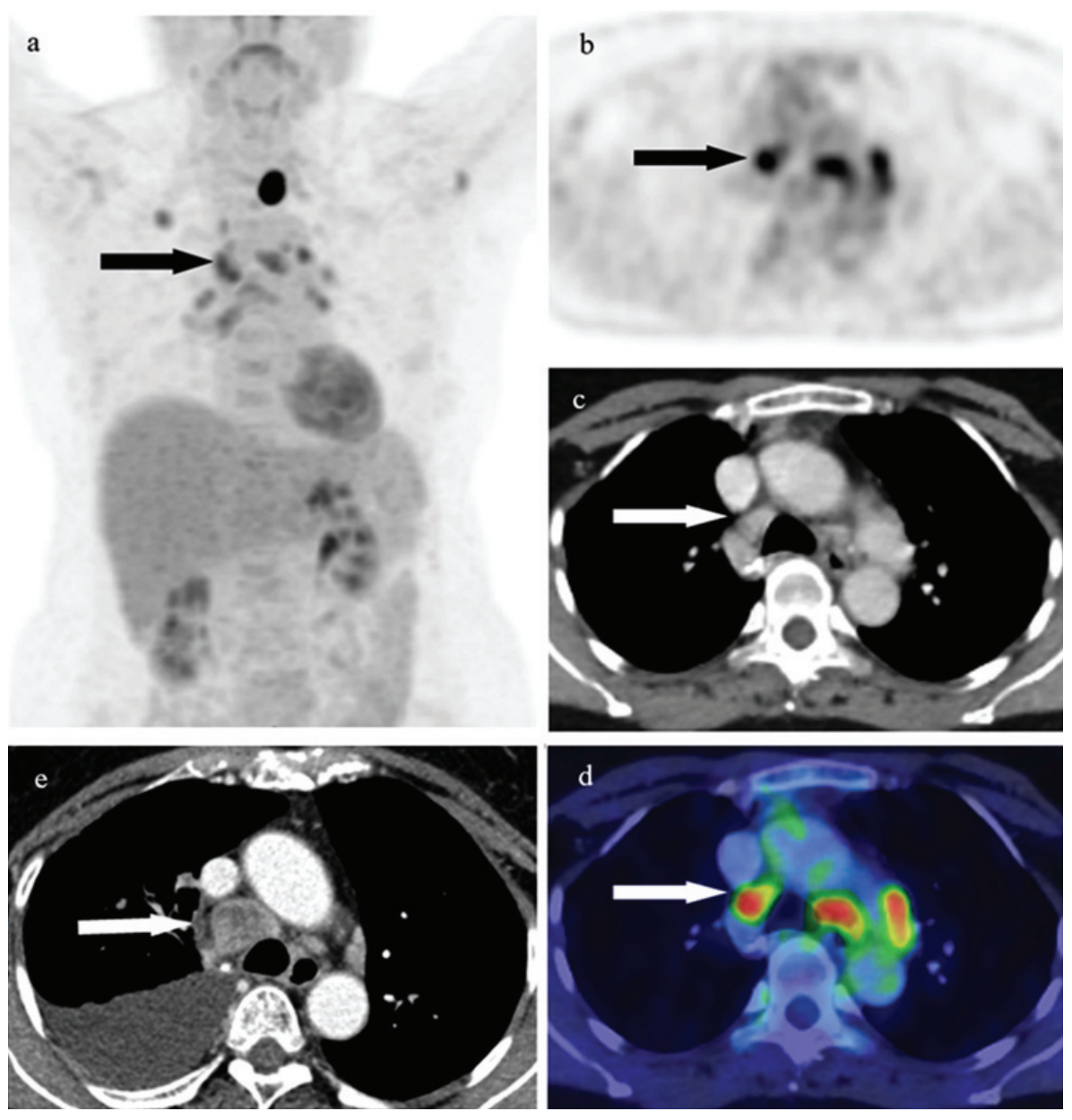

Figure 3 Metastatic lymph node: A 60-year-old female with stage IIIC colon cancer status post right hemicolectomy, with adjuvant chemotherapy presented with rising serum carcinoembryonic antigen. Fluorine-18 fluorodeoxyglucose positron emission tomography/computed tomography, showed multiple fluorodeoxyglucose-avid mediastinal lymph nodes. The most intense lymph node is at the right paratracheal region, with maximum standardized uptake values of 6.6 and lymph node-to-liver ratio of 2.2 (3a, 3b, 3c, 3d). A follow-up diagnostic chest computed tomography showed an increase in size of the lymph node from 1.2 to $2.5 \mathrm{~cm}(108 \%$ change from baseline), with internal necrosis (3e). The left thyroid nodule with intense fluorodeoxyglucose uptake is noted (3a).

\section{Discussion}

This study found that the prevalence of FDGavid mediastinal nodes in colorectal cancer patients in a tuberculosis-endemic area was as high as 68.8\%. One of the reasons that could be explained this finding is a high incidence of TB in Thailand; with an estimated total incidence of 150 per 100,000 in $2019 .^{13}$ However, this figure was much higher than a previous study from India that showed $37.3 \%$ FDG-avid mediastinal lymph nodes in abdomino-pelvic malignancies. ${ }^{14}$ Most of these patients with 
FDG-avid mediastinal nodes had multiple small lesions, bilaterally. The hilar and interlobar zones were the most common detected location. From the overall findings, benign reactive hyperplasia could be the most possible cause of mediastinal FDG uptake. Regarding the degree of FDG avidity, most the lymph nodes showed moderate uptake with median SUV $V_{\max }$ of 4.4. This finding was lower than the previous study, which found that the mean SUV $\max$ of the benign mediastinal lymph nodes; including, sarcoidosis and tuberculosis, was $5.02^{15}$. This was higher than the healthy subjects, which had a mean SUV $\max$ between 1.8-2.5. ${ }^{16,17}$ The degree of FDG-avid mediastinal nodes, by grading (visual assessment), could reliably differentiate lymph nodes with low, moderate, and high uptake, because of a significant difference of SUV $\max$ and LLR in each grade.

Older patients (age $\geq 50$ years) were found to have a significant association with FDG-avid mediastinal lymph nodes. This could be due to a higher incidence of TB as well as lower accessibility to immunization in the past. Hence, patients in this age group had a greater chance of getting infected by granulomatous disease than nowadays. Interestingly, there was no significant association between lung parenchymal lesion and mediastinal FDG uptake. This could be explained by an ongoing process of mediastinal lymphadenitis found in patients with old TB or inactive TB infection. ${ }^{17,18}$ Moreover, with the highest latent TB infection rate of $31.0 \%$ found in Southeast Asia ${ }^{19}$, several mediastinal FDG-avid nodes could be observed even in patients with absence of lung parenchymal lesions.

The significance of FDG-avid mediastinal nodes in $\mathrm{CRC}$ was validated in 32 patients, based on serial imaging or histopathology. Almost all the lymph nodes were benign in nature. This finding was consistent with the fact that mediastinal lymph node metastasis from CRC is extremely rare. ${ }^{5}$ Furthermore, the previous study also found that patients with $\mathrm{CRC}$, who had stable symmetrical and mild FDG uptake at bilateral hilar nodes on follow-up PET scans, were related to benign etiology. ${ }^{20}$ Only one patient had a $1.2-\mathrm{cm}$ metastatic node at the right paratracheal region, which showed high FDG uptake $\left(\mathrm{SUV}_{\max }\right.$ of 6.6 , LLR of 2.2). The previous case reports also demonstrated metachronous mediastinal node metastasis in CRC with

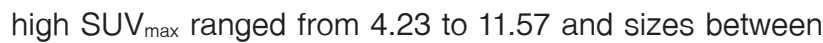
1.6 to $6 \mathrm{~cm} .^{5,6,8}$ The two possible routes for mediastinal node metastasis in CRC occurs via the thoracic duct from retroperitoneal nodes, and via the paravertebral venous plexus in patients with distant organ metastases. ${ }^{8}$

There were some limitations in our study. Firstly, the causes of FDG-avid mediastinal nodes could be only be identified in less than half of the patients, due to unavailable serial imaging or histopathology in some patients. Secondly, this being a retrospective study, other potential associated factors for presence of mediastinal FDG uptake; such as, history of TB infection or smoking, were difficult to evaluate. Further prospective studies, with a larger numbers of patients and longer follow-up duration is recommended to determine causes and associated factors for FDG-avid mediastinal nodes in this group of patients.

\section{Conclusion}

The prevalence of FDG-avid mediastinal nodes in patients with $\mathrm{CRC}$ in a tuberculosis-endemic area was significantly high. Most of the lymph nodes had moderate and high FDG avidity. However, almost all of them were benign in nature. Thus, interpretation of these findings should be carefully understood to avoid false-positive interpretation.

\section{Acknowledgement}

We would like to acknowledge Mr. Gurdeep Singh for editing the manuscript, via the Publication Clinic $\mathrm{KKU}$, Thailand. We are also grateful to Dr. Kaewjai Thepsuthammarat, Clinical Epidemiology Unit, for Biostatistical consultations. 


\section{Funding sources}

This research was supported by the Mining Data for International Publication Project, Faculty of Medicine, Khon Kaen University (MN63201).

\section{Conflict of interest}

There are no conflicts of interest.

\section{References}

1. Bray F, Ferlay J, Soerjomataram I, Siegel RL, Torre LA, Jemal A. Global cancer statistics 2018: GLOBOCAN estimates of incidence and mortality worldwide for 36 cancers in 185 countries. CA Cancer J Clin 2018;68:394-424.

2. Dekker E, Tanis PJ, Vleugels JLA, Kasi PM, Wallace MB. Colorectal cancer. Lancet 2019;394:1467-80.

3. Riihimäki M, Hemminki A, Sundquist J, Hemminki K. Patterns of metastasis in colon and rectal cancer. Sci Rep 2016;6: 29765.

4. Libson E, Bloom RA, Halperin I, Peretz T, Husband JE. Mediastinal lymph node metastases from gastrointestinal carcinoma. Cancer 1987;59:1490-3.

5. Toda K, Kawada K, Sakai Y, Izumi H. Metachronous mediastinal lymph node metastasis from ascending colon cancer: A case report and literature review. Int J Surg Case Rep 2017;41: 336-9.

6. Iwata T, Chung K, Hanada S, Toda M, Nakata K, Kato T, et al. Solitary bulky mediastinal lymph node metastasis from colon cancer. Ann Thorac Cardiovasc Surg 2013;19:313-5.

7. Kuba H, Sato N, Uchiyama A, Nakafusa Y, Mibu R, Yoshida K, et al. Mediastinal lymph node metastasis of colon cancer: eport of a case. Surg Today 1999;29:375-7.

8. Matsuda Y, Yano M, Miyoshi N, Noura S, Ohue M, Sugimura K, et al. Solitary mediastinal lymph node recurrence after curative resection of colon cancer. World J Gastrointest Surg 2014;6:164-8.

9. Jadvar H, Colletti PM, Delgado-Bolton R, Esposito G, Krause $\mathrm{BJ}$, lagaru $\mathrm{AH}$, et al. Appropriate Use Criteria for 18F-FDG PET/CT in Restaging and Treatment Response Assessment of Malignant Disease. J Nucl Med 2017;58:2026-37.

10. Yen RF, Chen KC, Lee JM, Chang YC, Wang J, Cheng MF, et al. 18F-FDG PET for the lymph node staging of nonsmall cell lung cancer in a tuberculosis-endemic country: is dual time point imaging worth the effort? Eur $\mathrm{J}$ Nucl Med Mol Imaging 2008;35:1305-15.

11. Lee SH, Min JW, Lee CH, Park CM, Goo JM, Chung DH, et al. Impact of parenchymal tuberculosis sequelae on mediastinal lymph node staging in patients with lung cancer. J Korean Med Sci 2011;26:67-70.

12. Chuchottaworn $C$, Thanachartwet $V$, Sangsayunh $P$, Than TZM, Sahassananda D, Surabotsophon M, et al. Risk Factors for Multidrug-Resistant Tuberculosis among Patients with Pulmonary Tuberculosis at the Central Chest Institute of Thailand. PLoS ONE 2015;10:e0139986.

13. Global Tuberculosis Report 2020 [monograph on Internet]. Geneva: World Health Organization; 2020 [cited 2021 Jan 16]. Available from: https://www.who.int/publications///tem/ 9789240013131

14. Abrar M, Bhoil A, Kashyap R, Bhattacharya A, Mittal B. Incidence and significance of FDG uptake in mediastinal lymph nodes in patients undergoing FDG PET/CT for abdomino-pelvic malignancies - An Indian perspective. J Nucl Med 2012;53: 1448.

15. Kumar A, Dutta R, Kannan U, Kumar R, Khilnani GC, Gupta $\mathrm{SD}$. Evaluation of mediastinal lymph nodes using 18F-FDG PET-CT scan and its histopathologic correlation. Ann Thorac Med 2011;6:11-6.

16. Kwan A, Seltzer M, Czernin J, Chou MJ, Kao CH. Characterization of hilar lymph node by $18 \mathrm{~F}$-fluoro-2-deoxyglucose positron emission tomography in healthy subjects. Anticancer Res 2001;21:701-6.

17. Kang WJ, Chung J-K, So Y, Jeong JM, Lee DS, Lee MC. Differentiation of mediastinal FDG uptake observed in patients with non-thoracic tumours. Eur J Nucl Med Mol Imaging 2004; 31:202-7.

18. Konishi J, Yamazaki K, Tsukamoto E, Tamaki N, Onodera Y, Otake $T$, et al. Mediastinal lymph node staging by FDG-PET in patients with non-small cell lung cancer: analysis of falsepositive FDG-PET findings. Respiration 2003;70:500-6.

19. Paton NI, Borand L, Benedicto J, Kyi MM, Mahmud AM, Norazmi $\mathrm{MN}$, et al. Diagnosis and management of latent tuberculosis infection in Asia: Review of current status and challenges Int $\mathrm{J}$ Infect Dis 2019;87:21-9.

20. Karam M, Roberts-Klein S, Shet N, Chang J, Feustel P. Bilateral hilar foci on 18F-FDG PET scan in patients without lung cancer: variables associated with benign and malignant etiology. J Nucl Med 2008;49:1429-36. 